\title{
CONHECIMENTOS E PRÁTICAS DE TRABALHADORES DA CONSTRUÇÃO CIVIL SOBRE PRÁTICAS DE PROMOÇÃO DA SAÚDE E ATENÇÃO À SAÚDE
}

\author{
KNOWLEDGE AND PRACTICES OF CONSTRUCTION WORKS ABOUT \\ HEALTH PROMOTION AND HEALTH CARE PRACTICES
}

\section{Fabiana Martins Sales de Melo \\ Graduada em Enfermagem e Fonoaudiologia Universidade Federal de Pernambuco. \\ E-mail: fabmsm@gmail.com}

\section{Fábia Alexandra Pottes Alves}

Doutora em Saúde Pública.

Departamento de Enfermagem da Universidade Federal de Pernambuco.

E-mail: fabia.alexandra@terra.com. br

Theresa Pricilla Calado de Barros Gonçalves

Graduanda em Enfermagem.

Universidade Federal de Pernambuco.

E-mail: theresapricilla@gmail.com

\section{Maria Ilk Nunes de Albuquerque}

Doutora em Serviço Social.

Departamento de Enfermagem

da Universidade Federal de

Pernambuco.

E-mail: nunesilk@gmail.com

\section{Maria Wanderleya de Lavor Coriolano- Marinus}

Doutora em Saúde da Criança e do Adolescente. Departamento de Enfermagem da Universidade Federal de Pernambuco.

E-mail: wandenf@yahoo.com.br

\section{Resumo}

Objetivos: identificar o conhecimento de trabalhadores da construção civil acerca da Política Nacional de Atenção à Saúde do Homem, traçar o perfil desses trabalhadores da construção civil quanto aos dados de identificação, condição socioeconômica e situação de saúde, acesso aos serviços de saúde para a prevenção e/ou tratamento de agravos e identificar os principais motivos e fatores culturais que interferem na busca pela atenção à saúde. Métodos: estudo transversal, com abordagem quantitativa. A coleta de dados foi realizada em empresas da construção civil, localizadas na cidade do Recife-PE. Foi aplicado um formulário estruturado, além da realização de uma atividade educativa que abordou questões sobre alimentação, uso de álcool e cigarro. Os dados foram analisados de forma descritiva e apresentados em tabelas, construídas pelo programa Epi Info 3.5.2. Resultados: Encontramos homens que na 
maioria classificam sua saúde como ótima/ boa $56(77,7 \%)$, a maioria faz uso do álcool $57(79,2 \%)$, não usam nenhum método de prevenção contra DST's45(62,5\%), procuram os serviços de saúde pela atenção especializada, pronto socorro/ ambulatórios 53(73,6\%) e desconhecem a Politica Nacional de Atenção Integral à Saúde do Homem. Quanto às informações de prevenção de doenças, quando as tinham não eram utilizadas no dia a dia. Conclusão: Evidencia-se uma população de trabalhadores jovens, que considera a saúde como boa, embora tenha sido evidenciado elevado percentual de uso de álcool, com situações de embriaguez no ambiente de trabalho. Conclusão: Faz-se necessária a intensificação das praticas dos serviços de saúde, com ações educativas de efeito para os homens.

Palavras-Chave: Saúde do Homem, Politica de Saúde, serviços de saúde.

\section{Abstract}

Objective: to identify the knowledge of construction workers on the National Health Care Human Policy, trace the profile of construction workers as the identification data, socioeconomic status and health status, access health services for the prevention and / or treatment of diseases and identify the main reasons and cultural factors that influence the quest for health care. Methods: cross-sectional study with a quantitative approach. Data collection was conducted in two companies of construction, located in the city of RecifePE. It was applied a structured form, besides the realization of an educational activity that addressed issues on food, alcohol and cigarettes. Data were analyzed descriptively and presented in tables, constructed by the
Epi Info 3.5.2 program. Results: We found that most men rate their health as excellent / good 56 (77.7\%), most uses of alcohol 57 (79.2\%), use no method of prevention DST's45 $(62.5 \%)$, seeking health services for specialized care, emergency room / outpatient 53 (73.6\%). By analyzing the covered population, there was ignorance of the National Policy for Integral Attention to Men's Health, as well as disease prevention information and when they were not used on a daily basis. Conclusion: it is necessary to intensify the practice of health services, educational activities with effect to men.

Keywords: Men's Health, Health Policy, health services

\section{Introdução}

Numa perspectiva de gênero onde o referencial de masculinidade aponta para padrões reconhecidos como característicos do ser homem, como viril, forte e provedor, negando inclusive, a existência de dor ou sofrimento e vulnerabilidades, reforçando a diferenciação com o feminino dito como frágil, contribui para maior visibilidade do processo saúde-adoecimento com relação à saúde dos homens. ${ }^{1}$

$\mathrm{Na}$ tentativa de reverter esse quadro ou modificar, em 2009, instituiu-se a Política Nacional de Atenção Integral à Saúde do Homem (PNAISH), que dispõe sobre a qualificação da atenção à saúde da população masculina na perspectiva de linhas de cuidado que resguardam a integralidade da atenção, propondo uma mudança na qualidade da assistência oferecida a essa população, bem como mudanças na visão cultural de cuidados aos homens na faixa etária de 20 a 59 anos de idade. $^{2}$ 
Os principais objetivos da Política são promover mudanças de paradigmas no que concerne à percepção da população masculina em relação ao cuidado com sua saúde, aumentando a expectativa de vida e reduzindo os índices de morbimortalidade dessa população, além do fortalecimento da assistência básica no cuidado com o homem, facilitando e garantindo o acesso e a qualidade de atenção necessária ao enfrentamento dos fatores de risco das doenças e dos agravos à saúde. ${ }^{2}$

Quando os indivíduos resistem na busca à promoção do autocuidado e são negligentes com as ações preventivas, não afetam apenas sua condição de saúde, mas oneram o sistema de saúde, que precisa direcionar o financiamento para os serviços de média e alta complexidade, que contempla internamentos e exames de alto custo. Sendo assim, é relevante que os homens procurem o serviço de saúde, pelo menos uma vez ao ano, além de serem necessários mecanismos locais para melhorar a assistência oferecida a essa população. $^{2}$

Porém, observa-se na prática dos serviços de saúde de atenção primária, uma baixa frequência da população masculina às ações de promoção da saúde e prevenção de doenças e agravos, por inúmeras razões das quais podem ser destacadas: desconhecimento e a falta de capacitação dos profissionais de saúde, a inexistência de mecanismos de captação e busca da população masculina por parte dos profissionais de saúde, a rotina de funcionamento do serviço de saúde no horário diurno, além de fatores relacionados ao âmbito cultural de gênero, como a falta de interesse dos homens no cuidado a sua saúde. ${ }^{3}$

O interesse da autora principal em investigar essa temática, partiu da vivência acadêmica na graduação em enfermagem, na qual observou que a demanda de usuários do sexo masculino em relação ao feminino era discrepante.
A partir da realidade observada no que se referia à deficiência de ações de saúde de promoção da saúde e prevenção de fatores de risco e agravos direcionados à população masculina, delimitou-se a questão de pesquisa: "Qual o conhecimento de trabalhadores da construção civil a cerca da política Nacional de Atenção Integral à Saúde do Homem? E qual o perfil dessa amostra em relação às variáveis sócioeconômicas e demográficas, fatores de risco para doenças e práticas de promoção à saúde?"

Os objetivos foram: identificar - conhecimento de trabalhadores da construção civil acerca da Politica Nacional de Atenção Integral à Saúde do Homem; traçar o perfil de homens trabalhadores de empresas da construção civil no que se refere a estado civil, condição socioeconômica, agravos à saúde, procura pelos serviços de saúde para a prevenção e/ ou tratamento de agravos e Identificar os principais motivos e fatores culturais que interferem na busca pela atenção à saúde.

\section{Método}

Pesquisa de natureza transversal com abordagem quantitativa ${ }^{4,5}$, realizada em duas empresas da construção civil localizadas na Região Metropolitana da cidade do Recife- PE. A escolha destes locais ocorreu por oferecerem, no seu quadro funcional, homens trabalhadores, nos mais diferentes cargos e por estes indivíduos estarem na faixa etária da PNAISH.

Foram incluídos trabalhadores da construção civil que estavam dentro da faixa etária da PNAISH, dos 20 aos 59 anos e que aceitaram participar na pesquisa e utilizavam os serviços do SUS.

As duas empresas possuíam um total de 79 homens que preenchiam aos critérios de inclusão da pesquisa. A coleta 


\section{Artigo Original}

foi realizada no canteiro de obras das empresas através duas visitas, sendo feita na primeira visita a aplicação do questionário estruturado, em uma sala previamente reservada com 72 participantes no total. Estes foram todos os que se encontravam nos canteiros de obras no momento de coleta de dados, ocorrendo duas recusas.

Na segunda visita das pesquisadoras, foi realizada uma ação educativa com orientações sobre hipertensão arterial, diabetes, dislipidemias, hábitos de alimentação, alimentação saudável. Esse momento contou com a participação de 38 homens que estavam no canteiro de obras. Após a ação educativa foram coletados dados da pressão arterial, da glicose pós prandial, peso, altura e realizado o cálculo de índice de massa corpórea (IMC).

Os dados foram digitados em um banco de dados do programa Epi-Info, versão 3.5.2. Para análise de dados não foram realizados testes de associação por se tratar de um tamanho amostral que não permitiu traçar um panorama analítico. Sendo assim, os dados foram analisados de forma descritiva e apresentados em tabelas.

Seguindo os preceitos éticos foi assinado o Termo de Consentimento Livre e Esclarecido (TCLE) após concordância verbal e esclarecimento dos objetivos deste estudo e sobre os critérios assegurados pela resolução 466/2012 do Conselho Nacional de Saúde.

O projeto foi aprovado pelo Comitê de Ética em Pesquisa do Centro de Ciências da Saúde da Universidade Federal de Pernambuco com o CAEE no 38714414.0.0000.5208.

\section{Resultados}

Dos indivíduos investigados, 70 (97,2\%), exerciam as funções das mais variadas (pedreiro, ajudante de pedreiro, marceneiro, eletricista, encanador, etc.) e apenas 2 (2,7\%), eram técnicos de segurança do trabalho. Trabalhadores de nível superior não foram entrevistados devido à ausência desses profissionais durante a fase de coleta de dados.

A tabela 1 apresenta os aspectos socioeconômicos e demográficos dos entrevistados.

Tabela 1 - Perfil de trabalhadores da construção civil segundo variáveis socioeconômicas e demográficas. $n=72$. Recife, 2015.

\begin{tabular}{lcc}
\hline Variáveis & $\mathbf{n}$ & $\%$ \\
\hline Faixa etária & 14 & \\
\hline 20 a 29 & 22 & $19,4 \%$ \\
30 a 39 & 19 & $30,6 \%$ \\
40 a 49 & 17 & $26,4 \%$ \\
50 a 59 & & $23,6 \%$ \\
Estado civil & 18 & \\
Solteiro & 24 & $25,0 \%$ \\
Casado & 4 & $33,3 \%$ \\
Viúvo & & $5,6 \%$
\end{tabular}




\section{Artigo Original}

União estável

22

$30,6 \%$

Outros

4

$5,6 \%$

\section{Tipo de residência}

Alugada

13

$18,1 \%$

Própria

53

$76,6 \%$

Outros

6

$8,3 \%$

№ de pessoas no domicílio

0 a 5

6 a 10

\section{Renda mensal}

Menos de 1 salário

De 1 a 3 salários

Mais de 3 salários
53

19

$73,6 \%$

$26,4 \%$

$\begin{array}{rr}1 & 1,4 \% \\ 63 & 87,5 \% \\ 8 & 11,1 \%\end{array}$

Fonte: Dado da pesquisa.

A tabela 2 apresenta os principais fatores de risco relacionados à saúde dos trabalhadores.

O cigarro era consumido por $21(29,2 \%)$ e destes $15(20,8 \%)$ consumiam de 1 a 20 cigarros por dia, 6(4\%) consumiam de 30 a 40 cigarros por dia. Em relação ao álcool, 57(79,2\%) dos trabalhadores consumiram na última semana e $15(20,8 \%)$ não consumiam em nenhum momento.

Quando questionados sobre terem sofrido algum acidente de trânsito no último ano, $12(7 \%)$ responderam que sim, dos quais $6(50,0 \%)$ precisaram ser hospitalizados. Desses acidentes, $11(91,7 \%)$, o tipo de veiculo foi à motocicleta.

Tabela 2 - Fatores de Risco à saúde de trabalhadores da construção civil. N= 72. Recife- PE, 2015.

\begin{tabular}{lcc}
\hline Variáveis & $\mathbf{n}$ & $\%$ \\
\hline Uso de cigarro & 21 & \\
Sim & 51 & $29,2 \%$ \\
Não & 15 & $70,8 \%$ \\
1 a 20 cigarros & 6 & $20,8 \%$ \\
30 a 40 cigarros & & $4 \%$ \\
Bebida alcoólica na última semana & 57 & \\
Sim & 15 & $79,2 \%$ \\
Não & & $20,8 \%$
\end{tabular}




\begin{tabular}{lll} 
Acidentes de transito no último ano & 12 & $16,9 \%$ \\
Sim & 59 & $83,1 \%$ \\
Não & & \\
Doenças prévias & 19 & $26,4 \%$ \\
Sim & 53 & $73,6 \%$ \\
Não & & \\
\hline
\end{tabular}

Fonte: Dados da pesquisa.

Na tabela 3, apresentam-se questões relacionadas à promoção da saúde e prevenção de doenças e agravos, constatando-se que em relação à autoclassificação de sua saúde a maioria, 56 (77,8\%), classificou sua saúde como ótima ou boa e apenas 5 (7,0 \%) classificaram como ruim ou péssima.

Os métodos anticoncepcionais definitivos foram os mais encontrados, referidos por $22(45,8 \%)$ (laqueadura, histerectomia). Em $8(16,7 \%)$, as companheiras utilizavam a pílula anticoncepcional, $10(20,8 \%)$ usavam a camisinha masculina, $6(12,5 \%)$, as companheiras faziam uso do anticoncepcional injetável. Quando indagados sobre a prevenção contra as doenças sexualmente transmissíveis (DST), utilizando camisinha masculina, 27 (37,5\%) afirmaram sua utilização, embora na pergunta anterior sobre concepção apenas 10 referiram a utilização desse método.

A medição da pressão arterial no último ano foi realizada por $46(63,9 \%)$. Em relação à glicose, apenas $15(20,8 \%)$ afirmaram ter medido no último ano. O tipo de serviço de saúde mais utilizado diante de necessidades em saúde foram o pronto socorro por $30(41,7 \%)$, a Unidade de Saúde da Família por 18 (25,0\%) e os hospitais por 17(23,6\%).

Quanto ao conhecimento por PNAISH, 52(72,2\%) afirmaram não ter conhecimento sobre a existência de tal Política. No acesso à informação sobre prevenção de doenças, a maioria, $37(51,4 \%)$ dos entrevistados afirmou ter esse conhecimento, sendo o Agente Comunitário de Saúde uma relevante fonte de informações.

Tabela 3 - Práticas de promoção da saúde e prevenção em trabalhadores da construção civil. $n=72$. Recife $-P E, 2015$.

\begin{tabular}{lrc} 
Variáveis & \multicolumn{1}{c}{$\mathbf{n}$} & $\%$ \\
Auto-classificação da saúde & & \\
Ótima & 13 & $18,1 \%$ \\
Boa & 43 & $59,7 \%$ \\
Ruim & 4 & $5,6 \%$ \\
Péssima & 1 & $1,4 \%$ \\
Regular & 11 & $15,3 \%$ \\
Uso de métodos anticoncepcionais & & \\
Pílula (companheira) & 8 & $16,7 \%$ \\
Camisinha masculina & 10 & $20,8 \%$
\end{tabular}




\section{Artigo Original}

$\begin{array}{lrc}\text { Injeção (companheira) } & 6 & 12,5 \% \\ \text { Nenhum método } & 2 & 4,2 \% \\ \text { Outros } & 22 & 45,8 \%\end{array}$

Prevenção contra DST

$\begin{array}{lll}\text { Sim } & 27 & 37,5 \% \\ \text { Não } & 45 & 62,5 \%\end{array}$

Medida da PA no último ano

Sim $46 \quad 63,9 \%$

Não

26

$36,1 \%$

Medida da glicose no último ano

Sim 15

$20,8 \%$

Não

57

$79,2 \%$

Serviço de saúde que mais procura

Ambulatórios

$2 \quad 2,8 \%$

Unidade de Saúde da Família

$18 \quad 25,0 \%$

Pronto Socorro

30

$41,7 \%$

Hospital

17

$23,6 \%$

Outros

4

$5,6 \%$

\section{Conhece sobre a PNAISH}

Sim 20

$27,8 \%$

Não

57

$72,2 \%$

\section{Acesso à informação sobre prevenção de} doenças

$\begin{array}{lll}\text { Sim } & 37 & 51,4 \% \\ \text { Não } & 35 & 48,6 \%\end{array}$

Fonte: Dados da pesquisa.

Após a coleta de dados através do questionário, foi realizada uma ação educativa abordando a importância da alimentação adequada na saúde, os tipos de alimentos que influenciam diretamente na alteração do padrão da pressão arterial, glicemia e dislipidemia., Apenas 38 indivíduos compareceram a essa atividade. Na avaliação do estado de saúde dos participantes foi realizada a mensuração da PA, Glicemia Pós Prandial, Peso e Estatura. Com os dados, peso e altura foram calculados o IMC. Os dados referentes à essa etapa encontram-se descritos na Tabela 4.

Dos 38 participantes, 32(84,3\%) apresentavam PA em estágio Pré Hipertensão, 5 (13,2\%), apresentavam estágio 1 de hipertensão e apenas 1(2,6\%), apresentava PA dentro do padrão de normalidade. Desses 38 entrevistados, nenhum fazia uso de medicação anti-hipertensiva ou medicação para controle da glicose. 


\section{Artigo Original}

Com a verificação da glicemia pós prandial, identificou-se que $18(47,4 \%)$ estavam acima do padrão de $140 \mathrm{mg} / \mathrm{dl}$, indicando intolerância à glicose. Ao calcular o IMC dos entrevistados identificou-se que, $22(57,9 \%)$, estavam com sobrepeso e $6(15,8 \%)$ com obesidade grau I.

Tabela 4 - Avaliação nutricional e mensuração de pressão arterial, glicemia, peso e estatura (IMC) em trabalhadores da construção civil. N=38. Recife, 2015.

\begin{tabular}{lrc}
\hline Variáveis & N & $\%$ \\
\hline Peso & 7 & $18,4 \%$ \\
50 a 70 & 23 & $60,5 \%$ \\
71 a 90 & 8 & $21,1 \%$ \\
91 a 120 & & \\
\hline Pressão Arterial & 1 & $2,6 \%$ \\
\hline $120 /<80$ & 32 & $84,3 \%$ \\
$120-139 / 80-89$ & 5 & $13,1 \%$ \\
$140-159 / 90-99$ & & \\
\hline Glicemia & 20 & $52,6 \%$ \\
73 a $140 \mathrm{mg} / \mathrm{dl}$ & 18 & $47,4 \%$ \\
144 a $166 \mathrm{mg} / \mathrm{dl}$ & & \\
IMC & 10 & $26,3 \%$ \\
18,5 a 24,99 & 22 & $57,9 \%$ \\
25 a 29,99 & 6 & $15,8 \%$ \\
\hline 30 a 34,99 & & \\
\hline
\end{tabular}

Fonte: Dados da pesquisa.

\section{Discussão}

Os participantes, em sua maioria, eram casados ou em união estável com renda entre 1 a 3 salários mínimos, morando em casa própria, que abrigavam, de 1 a 5 moradores, e dentro de uma faixa etária predominante de 30 a 39 anos, perfil que se enquadra com o já descrito em outras pesquisas sobre a população masculina.

Em pesquisa com homens com idade acima de 21 anos, alfabetizados, o perfil encontrado, estava diante de homens inseridos no mercado de trabalho, situação que muitas vezes impede a busca por serviços de atenção a saúde, devido à incompatibilidade de horários dos serviços de saúde com o horário de trabalho, o que na visão dos mesmos, os impedem de ausentar-se do trabalho, pelo risco de perdê-lo, para cuidar de sua saúde. ${ }^{6}$

$\mathrm{Na}$ busca dos principais fatores de risco à saúde, o álcool e o cigarro foram os principais problemas identificados. $O$ álcool era consumido por $79,2 \%$ dos pesquisados e no momento da entrevista e no ambiente de trabalho, não foi difícil observar que existiam participantes 
alcoolizados. Além do consumo do álcool, em excesso, ser prejudicial à saúde, o cenário de trabalho da construção civil, por si só, apresenta vários riscos de acidentes de trabalho e com indivíduos alcoolizados, esse risco pode ser potencializado. ${ }^{7}$

O relatório do Status Mundial de Álcool e Saúde da Organização Mundial de saúde (OMS) de 2011 ${ }^{8}$, afirma que o consumo de álcool é o terceiro maior fator de risco para doenças e incapacidade no mundo e o maior deles em países de renda média.

Outro fator risco à saúde encontrado foi o consumo do cigarro. O tabagismo passivo é a 3a causa de morte evitável no mundo e o maior responsável pela poluição em ambientes fechados. A maioria não consome o cigarro, mas são fumantes passivos, pois convivem com os fumantes ativos. Os fumantes passivos correm riscos assim como os que fumam, são os tabagistas passivos e em ambientes fechados, o risco é 30\% maior de desenvolver um câncer de pulmão, $25 \%$ maior de desenvolverem doenças cardiovasculares, além de asma, pneumonia, sinusite, dentre outras. ${ }^{9}$

No ultimo ano, 12 (7\%) dos entrevistados sofreram acidente de trânsito, dos quais $6 \quad(50,0 \%)$ ficaram internados e $11(91,7 \%)$, foram causados por motocicletas. A PNAISH aponta alguns indicadores de morbidade e mortalidade que se destacam na população masculina, e os acidentes de transito estão enquadrados nas causas externas que atingem esse grupo populacional na faixa etária dos 25 a 59 anos. ${ }^{10}$

Quanto à autoclassificação da saúde, a maioria, $56(77,7 \%)$, referiu como ótima ou boa, relatando "não sentiram nada", e ainda, muitos indivíduos tinham a percepção de que apresentavam algum sintoma, mas dificilmente aceitavam ou reconheciam a necessidade de procurar um serviço de saúde. Esses dados são corroborados por estudo ${ }^{10}$ que pontua que os homens não reconhecem suas necessidades de saúde no âmbito preventivo, sendo assim cultivam a ideia que não adoecem e só procuram a atenção quando as doenças estão instaladas.

A doença é considerada sinal de fragilidade, e os homens não reconhecem como algo inerente à sua condição biológica, por isso o julgamento da invulnerabilidade, ficando expostos a condições de risco. ${ }^{11}$

Enquanto há preocupação com o aumento da família, dentre os entrevistados, percebe-se nas respostas quanto ao uso de métodos anticoncepcionais, não vêm com medidas de proteção contra as DST, as quais estão aumentando significativamente no Brasil e são consideradas um problema de saúde pública. As estimativas da OMS é que, a cada ano surgem 330 milhões de novos casos de DST, aproximadamente, 1 milhão a cada dia; por não serem de notificação compulsória, o país não têm dados precisos, à exceção da AIDS e Sífilis. ${ }^{12,13}$

A epidemia da AIDS motivou a inserção do homem nos debates sobre saúde sexual, passando a se falar sobre prevenção e mudança comportamental a ser adotada na relação sexual. O uso da camisinha, amplamente divulgado como método mais eficaz na prevenção de DST/AIDS é o que vem mostrando ao longo dos anos, porem sua adesão ainda apresenta desafios ao controle das DTS/AIDS. ${ }^{14}$

Dos participantes da pesquisa 46 $(63,9 \%)$, mediram a PA no último ano em algum momento e apenas 15 (20,8\%), mediram a glicose no último ano. A saúde masculina ainda está vinculada quase exclusivamente ao exame preventivo de câncer de próstata, deixando de lado a prevenção de outros fatores de risco em homens jovens. ${ }^{6}$

A maioria não buscava os serviços de atenção primária, sendo mais frequentes a busca como serviço de "porta de entrada" o pronto socorro para $41,7 \%$ e os hospitais para $23,6 \%$. Esse resultado semelhante aos dados de Vieira et al. ${ }^{11}$, ressaltam, 
que a população masculina acessa o sistema de saúde por meio de atenção especializada, corroborando a necessidade do fortalecimento e qualificação da atenção primária.

Pereira; Nery ${ }^{15}$ referem a necessidade de uma reflexão sobre a necessidade de reestruturação da relação entre homens e serviços de saúde no sentido de promover qualidade de vida à população masculina. Fagundes et $a^{16}$ relatam a unanimidade entre os profissionais de saúde quando afirmam que os homens, além de menos presentes e assíduos no serviço de saúde, também são mais resistentes aos convites para irem ao serviço, assim como o índice de faltas e a não adesão aos tratamentos são os maiores por parte dos homens.

Mesmo diante da morbimortalidade masculina, a busca pelos serviços de saúde ainda é pequena quando comparada à busca feminina, deixando claras as barreiras culturais e o medo da descoberta de uma doença grave, e como um fator de proteção, preferem não ter conhecimento. ${ }^{11,12,15}$

Quanto ao acesso a informações sobre prevenção de doenças, um grande percentual dos entrevistados não tem acesso a essas informações, o que indica a necessidade urgente de oferecer a essa população ações de promoção a saúde e prevenção de doenças. A PNAISH enfatiza que um aspecto ainda determinante no baixo acesso dos homens aos serviços de saúde, deve-se ao fato de que, os serviços e as estratégias de comunicação não tem privilegiado esse público. ${ }^{16}$

Cavalcanti et $a l^{6}$ ao estudarem as necessidades de homens e os principais obstáculos para uma atenção integral por parte da equipe de saúde, apontam como obstáculos referenciados pelos homens, a vergonha de se expor e a impaciência enfrentada durante a espera por atendimento, a inexistência de tempo para dedicar a sua saúde, atribuída ao regime de trabalho e a falta de resolutividade das necessidades de saúde. Os entrevistados apontaram como estratégias de enfrentamento a essas dificuldades, a necessidade de um acolhimento adequado pelo serviço de saúde, a comunicação adequada, através de informações e a formação de vínculo, através da estabilidade profissional e da visita domiciliar, evidenciando-se que os profissionais que compõem a ESF precisam se apropriar da estratégia de humanização nas suas práticas em saúde.

Dos entrevistados, a grande maioria foi identificado com PA alterada na realização da aferição, $(84,3 \%$ em estágio Pré Hipertensão e 13,1\% em estágio 1 de Hipertensão). Nenhum deles fazia uso de medicação anti-hipertensiva. A VI Diretriz Brasileira de Hipertensão ${ }^{17}$ caracteriza a HAS como síndrome crônica multifatorial, com prevalência preocupante, que atinge aproximadamente $25 \%$ da população mundial e com previsão de crescimento chegando aos $60 \%$ até 2025 e com maior prevalência no sexo masculino. O Ministério da Saúde, classifica a pressão Arterial como Normal $(<120<80 \mathrm{mmHg})$, Pré-hipertensão ( 120-139/80-89mmHg), Hipertensão em Estágio 1 (140-159/90-99mmHg) e Estágio 2 $(>160>100 \mathrm{mmHg})$. Com isso, é necessário - controle dos níveis pressóricos, pois esses níveis acima de $115 / 75 \mathrm{mmHg}$ elevam os riscos de mortalidade por doença cardiovascular. ${ }^{17,18}$

Esses dados apontam a necessidade de ações que possam beneficiar esse publico, minimizando os efeitos da hipertensão arterial a partir de mudanças comportamentais voltadas para a alimentação saudável, pratica de atividade física regular e aconselhamento ao não uso do fumo e álcool.

Mesmo com a identificação de que a maioria dos entrevistados estava com valores da glicemia pós prandial, dentro da normalidade, destaca-se o percentual de $47,4 \%$ desses trabalhadores que se 
encontravam acima do nível de tolerância $140 \mathrm{mg} / \mathrm{dl}$, indicando intolerância à glicose.

Esses resultados preocupam, sobretudo pelo fato das duas doenças (HA e DM) serem fatores de risco para doenças cardiovasculares.

Em relação ao parâmetro de sobrepeso/obesidade, identificou-se que a maioria 22 (57,9\%), encontrava-se com o índice acima do normal e em grau 1 de obesidade. Esses dados caminham para lado inverso do que os participantes acreditam, já que a maioria afirma que sua saúde é ótima/boa, corroborando com Ribeiro et $a l^{19}$, quando afirmam que a maioria dos homens desconhece sobre sua saúde, por negar a existência de pelo menos uma doença ao longo de sua vida, levando-o a procurar apenas os meios curativos e não os preventivos.

\section{Conclusão}

Esta pesquisa foi significativa embora não aplicada a todos os trabalhadores da construção civil, deu a oportunidade de ouvir um pequeno grupo de homens que têm o SUS como o único serviço de saúde.

Percebeu-se que poucos sabiam da existência da PNAISH, assim como informações sobre prevenção de saúde e quando as tinham não eram utilizadas no dia a dia, pôde-se conhecer sobre as dificuldades da busca, a falta de conhecimento sobre medidas de promoção da saúde, prevenção e assistência à saúde. Nesse contexto, faz-se necessário a intensificação das praticas dos serviços de saúde, com ações educativas de efeito, uma vez que essa pesquisa mostrou alterações importantes nos parâmetros clínicos.

As equipes da atenção básica tem a possibilidade de captar esses homens, porém esbarram nos entraves como a falta de ações contínuas, que são prejudicadas pela falta de capacitação de profissionais de saúde, falta de organização de horários de funcionamento compatíveis com o trabalho da população masculina.

A PNAISH, desde sua criação e implantação, ainda não conseguiu inserir o homem no contexto de saúde. Torna-se evidente que, a PNAISH precisa ir muito além para se consolidar na prática dos serviços de saúde, portanto, identifica-se a necessidade da realização de ações educativas de caráter continuado, de um plano estratégico que identifique as principais queixas do público alvo da política, e, que os profissionais de saúde sejam capacitados para o atendimento especifico a esse grupo populacional a partir de parcerias intersetoriais.

\section{Referências}

1. Machin R et al. Concepções de gênero, masculinidade e cuidados em saúde: estudo com profissionais de saúde da atenção primária. Ciência \& Saúde Coletiva, 2011; 16(11):4503-12.

2. Brasil. Portaria no1.944, de 27 de agosto de 2009. Instituto no âmbito do Sistema de Único de Saúde (SUS). Politica Nacional de Atenção Básica à Saúde do Homem. Diário Oficial da União, Brasília; 28 de ago 2009

3. Leal AF, Figueiredo WS, Nogueira da Silva GS. O percurso da Politica Nacional de Atenção Integral à Saúde do Homem (PNAISH), desde a sua formulação até sua implementação nos serviços públicos locais de atenção à saúde. Ciência \& Saúde Coletiva, 2012; 17(10):2607-16. 
${ }^{4}$. Gunther H. Pesquisa qualitativa versus Pesquisa quantitativa: Esta é a questão?. Psic. Teor e Pesq., 2006; 22(2):201-10.

${ }^{5}$. Turato ER. Métodos qualitativos e quantitativos na área da saúde: definições, diferenças e seus objetos de pesquisa. Rev Saude Publica, 2005; 39(3):507- 14.

${ }^{6}$. Cavalcanti JRD et al. Assistência Integral a saúde do Homem: necessidades, obstáculos e estratégias de enfrentamento. Esc Anna Nery, 2014; 18(4): 628-34.

7. Pessoa LL. Riscos de acidente de trabalho na construção civil . Revista Jus Navigandi 2014; 19:3871. Disponível em: <http://jus.com.br/artigos/26605>.

${ }^{8}$. Morais MLS, Rosa TEC, Moraes CL de. Prevalência do consumo abusivo de álcool em homens no estado de São Paulo: apontamentos para uma abordagem do alcoolismo na Atenção Básica à Saúde. BIS, Bol. Inst. Saúde (Impr.)2012; 14(1):73-9.

9. Instituto Nacional de Câncer. Um grave problema de saúde pública. 2007. Disponível: em: <http://www1.inca.gov.br/inca/Arquivos/t_Tabagismo.pdf >. Acesso em: 10 jun 2015.

10. Moreira RLSF, Figueiredo MLF, Fontes WD de, Barboza TM. Dificuldades de inserção do homem na atenção básica a saúde: a fala dos enfermeiros. Esc. Anna Nery 2014; 18(2):615621.

11. Vieira LCS, Figueiredo MLF, Sales RLUB, Lopes WMPS, Avelino FVD. A política nacional de saúde do homem: uma reflexão sobre a questão de gênero. Revista Enfermagem em Foco, 2011; 2(4): 215-17.

12. Mozer IT, Corrêa AC de P. Implementação da Politica Nacional de Saúde do homem: o caso de uma capital Brasileira. Esc. Anna Nery, 2014; 18(4):578-85.

13. Siqueira FAA, Silva SO, Almeida MSB, Lima TNB, Prest Bisneto F. Promoção e Prevenção à Saúde Sexual Masculina: Desafios das Equipes de Saúde da Família José Pinheiro. Rev. Brasileira de Ciências da Saúde 2011; 15(2):191-200.

${ }^{14}$. Rodrigues MJ. Doenças Sexualmente Transmissíveis (DTS) na Adolescência. Nascer e Crescer 2010; 19(3):200.

${ }^{15}$. Pereira LP, Nery AA. Planejamento, gestão e ações à saúde do homem na estratégia de saúde da família. Esc. Anna Nery 2014; 18(4):635-43.

${ }^{16}$. Fagundes PB. Saúde do homem: novas tecnologias e estratégias de prevenção das DST/HIV/ AIDS e Hepatites Virais. 2011 [Graduação]. Curso de Enfermagem, Faculdade de Enfermagem da Universidade Federal de Goiás, Goiás; 2011.

17. Brasil. VI Diretrizes Brasileiras de Hipertensão. Revista Brasileira de Hipertensão, 2010; 17(1):1-69.

18. Araújo JL, Paz EPA, Moreira TMM. Hermenêutica e o cuidado de saúde na hipertensão arterial realizado por enfermeiros na Estratégia Saúde da Família. Esc Anna Nery, 2010; 14(3):560-566.

19. Ribeiro MS. et al. Conhecimento de Homens Trabalhadores sobre sua Saúde na faixa etária de 20 a 40 anos em uma empresa de alimentos no Município de Araguaína ? TO. Revista Cientifica do ITPAC 2012; 5(3). 